\title{
Associations among Discharge Opioid Prescribing and Inpatient Postpartum Opioid Usage after Delivery
}

\author{
Ashley Veade, MD ${ }^{1}$ Tyler McKinnish, MD ${ }^{1}$ Ebony Carter, MD, MPH² Adam Lewkowitz, MD, MPHS 3 \\ ${ }^{1}$ Department of Obstetrics and Gynecology, Washington University \\ of St. Louis, St. Louis, Missouri \\ Address for correspondence Ashley Veade, MD, 4901 Forest Park \\ 2 Division of Maternal Fetal Medicine, Washington University of St. \\ Avenue, St. Louis, MO 63108 (e-mail: aveade@wustl.edu). \\ Louis, Saint Louis, Missouri \\ ${ }^{3}$ Division of Maternal Fetal Medicine, Women and Infants Hospital, \\ Alpert Medical School of Brown University, Providence, Rhode Island \\ Am J Perinatol Rep 2020;10:e275-e280.
}

\begin{abstract}
Keywords

- opioids

- vaginal delivery

- cesarean delivery

- postpartum

Objective The aim of the study is to identify an association between inpatient opioid consumption and prescription at discharge after vaginal delivery (VD) and cesarean delivery (CD).

Methods This retrospective cohort study included women with an active inpatient opioid order after VD or CD between July and October of 2018 at a single academic tertiary hospital. Women with opioid use disorder, 3rd or 4 th degree lacerations, wound complications, and peripartum hysterectomy were excluded. Oxycodone 5-mg (mg) tablets consumed postpartum and prescribed at discharge and sociodemographics were recorded. Primary outcome was the number of oxycodone 5-mg tablets prescribed at discharge. Outcomes were analyzed using multivariable logistic regression between quartiles of inpatient opioid consumption.

Results A total of 437 patients were included: 169 patients underwent VD, and 268 underwent $C D$. For VD and $C D$, women in the highest quartile of inpatient opioid consumption were more likely Black compared with the lowest quartile $(p=0.006$ and $p=0.004$, respectively). No association existed between inpatient opioid use and number of tablets prescribed at discharge for VD or CD (odds ratio [OR] 0.22 [95\% confidence interval or $\mathrm{Cl} 0.02-2.17$ ] and $\mathrm{OR} 1.04$ [95\% Cl 0.85-1.32], respectively). Conclusion The number of opioid tablets prescribed at discharge had no association with inpatient postpartum consumption after VD or CD.
\end{abstract}

Of the 63,000 people who died from drug overdose in 2016, approximately $66 \%$ involved opioids, ${ }^{1}$ and $40 \%$ of opioid overdoses were from prescription opioids. ${ }^{2}$ This demonstrates the role of medical providers in the current opioid epidemic. Each year in the United States, there are over 3 million vaginal deliveries and 1.3 million cesarean sections, and up to 27 and $75.5 \%$ of women filled an opioid prescription after delivery at discharge for vaginal deliveries and cesarean

received

May 21, 2020

accepted after revision

July 20, 2020
DOI https://doi.org/

10.1055/s-0040-1716906. ISSN 2157-6998. sections, respectively. ${ }^{2}$ As such, opioid prescribing for postdelivery pain management is one of the most common sources of prescribed opioids nationally. ${ }^{3,4}$ It is especially relevant for the practicing obstetrician as 1 in 300 opioid naïve patients becomes long-term opioid users. ${ }^{5}$ There are no national guidelines for outpatient opioid prescribing, with an optimal amount of post-delivery opioids not yet identified for the practicing obstetrician. However, recently published
Copyright $\odot 2020$ by Thieme Medical Publishers, Inc., 333 Seventh Avenue, New York, NY 10001, USA. Tel: +1(212) 760-0888.
License terms

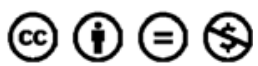


Committee Opinion from ACOG (American College of Obstetricians and Gynecologists) recommends utilizing a multimodal stepwise approach to limit opioids administered especially after cesarean delivery (CD). ${ }^{5}$ In other surgical specialties, the Centers for Disease Control and Prevention (CDC) recommends 3 days or less duration of treatment for acute pain with an opioid, with a maximum duration of 7 days. ${ }^{6}$ Applying these recommendations to postpartum patients would suggest that obstetric patients should experience the majority of their opioid consumption setting inpatient after delivery, ${ }^{7}$ and that obstetricians overprescribe opioids after cesarean section and vaginal delivery (VD). ${ }^{3,4,8}$

One strategy to optimize post-delivery opioids prescription is to tailor the number of opioid tablets prescribed according to inpatient usage to ensure patient pain is appropriately treated without providing excess opioids. Indeed, quantifying inpatient opioid consumption after vaginal and $\mathrm{CD}$ could be helpful in guiding the amount of tablets for postdischarge opioid prescribing. However, there is little data evaluating inpatient opioid usage after VD and cesarean sections in relation to the amount of opioids prescribed at discharge. One prospective observational cohort study of 179 women did suggest little association between inpatient opioid usage and discharge prescribing amount after $\mathrm{CD} .{ }^{9}$ Another retrospective study of 12,326 women highlighted approximately $45.7 \%$ of women after VD who received an opioid at time of discharge used $0-\mathrm{mg}$ morphine equivalents (MMEs) on final day of discharge. ${ }^{10}$ Our study adds to the previous studies and more closely examines total inpatient postpartum opioid consumption. This study aims to evaluate whether there is an association between the amount of tablets consumed during the entire inpatient postpartum admission and the amount of tablets prescribed at hospital discharge after both VD and cesarean section.

\section{Materials and Methods}

This retrospective cohort study included women who had an active inpatient opioid order in the electronic medical record (EMR) after a VD or cesarean section between July, 2018 and October, 2018 at a single academic tertiary care center. The dates selected were reflective of initiation of a central electronic medical record at our institution, allowing for identification of eligible patients. Women were excluded if they had a history of opioid usage prior to delivery, third- or fourth- degree laceration at time of delivery, wound complications, and peripartum hysterectomy. Opioid usage prior to delivery was defined as a known diagnosis of opioid use disorder or receipt of more than one opioid prescription during pregnancy, as identified per review of our comprehensive EMR, which integrates inpatient and outpatient care at our institution. Wound complications were defined as wound infections, seromas, or hematomas requiring surgical or medical intervention. The Washington University in St. Louis Human Research Protection Office approved this study (IRB \# 201901055).

The medical records of all women who delivered within the study period were reviewed to identify eligible women.
Participant clinical and sociodemographic information was extracted from qualifying subjects. Sociodemographic data included age, race, body mass index (BMI), insurance payer, length of post-delivery stay in days, and the discharge opioid prescriber (intern, resident, or attending). Race was reported as "Black" versus "White" as recorded in our EMR; the minority of women who were neither Black nor White were excluded given low prevalence at our institution.

The number of opioid tablets consumed post-delivery while inpatient and prescribed at discharge were recorded. At our institution, 5-mg oxycodone tablets are the most prescribed inpatient opioid: 94.6 and $97.0 \%$ of prescriptions are 5-mg oxycodone for VD and cesarean section, respectively. Therefore, other forms of opioids were converted to MME before being converted to 5-mg oxycodone tablets for ease of comparison. The primary outcome of this study was the number of oxycodone 5-mg tablets prescribed at discharge after delivery, stratified by mode of delivery.

At our academic institution, our hospital obstetrical practice consists of both private and nonprivate patients. Routine postpartum care universally involves nurse practitioners and residents. It is routine to order oxycodone 5-mg tablets for post- $C D$ as needed for pain in postpartum order sets, while not ordered for after VD. Inpatient postpartum opioids after VD are ordered at physician discretion. Additionally, acetaminophen and ibuprofen are routinely ordered as scheduled after $C D$, and as needed after VD for first line pain control. If any opioid is prescribed at discharge, both acetaminophen and ibuprofen are prescribed as first line agents, and patients counseled on pain management. However, there are no guidelines or recommendations for the amount of opioid tablets prescribed at discharge after VD. For CD, there are no formal guidelines or recommendations either, but an arbitrary number is selected by the discharging physician. A "common practice" of discharge is 5-mg oxycodone tablets after $C D$ ranging from 10 to 30 tablets. Ultimately, all discharge opioid prescriptions are at the discretion of discharging physician. Discharge provider includes either a resident physician, nurse practitioner, or attending physician, with most commonly prescribing provider a nurse practitioner or resident physician.

Participants were stratified by mode of delivery into quartiles by inpatient opioid usage. Demographic and baseline clinical data were compared between women who had the highest versus lowest quartile of inpatient opioid usage using a chi-square test or Fisher's exact test for categorical variables, as appropriate, and the Student's $t$-test or Mann-Whitney $U$-test for continuous variables. Multivariable regression models stratified women by inpatient opioid use and mode of delivery and analyzed primary outcomes, which were the number of opioid tablets prescribed at discharge. For women who had VD, though there were differences in age, maternal BMI and race, obstetric lacerations, and obstetric complications (such as postpartum operating room procedure, hemorrhage, episiotomy, or cervical laceration) between the study groups, multivariable analyses were not performed due to small numbers in the study population. Sensitivity analyses were then performed excluding women who underwent bilateral tubal 
ligation (BTL) after VD. For women who had CD, multivariable logistic regression was adjusted for race and day of postpartum discharge. Sensitivity analyses were then performed excluding women who were discharged at or after postoperative day five after CD.

\section{Results}

In total, 437 patients met inclusion criteria. Of these, 169 patients underwent VD, and 268 underwent CD.

\section{Vaginal Delivery}

Among the 169 included patients, the median number of oxycodone 5-mg tablets consumed as an inpatient was one tablet (interquartile range [IQR] 0, 3). However, 30.1\% $(51 / 169)$ of patients received a prescription for oral opioids on discharge. The median number of tablets prescribed at discharge among patients who received a prescription was 15 (IQR 10, 20). Delivery, nearly one-third ( $n=16 ; 32 \%)$ received a postpartum opioid prescription, with a median of 10 tablets (IQR 5, 16.25).

In - Table 1, sociodemographic characteristics of the top quartile of post-VD inpatient opioid users-who consumed greater than or equal to three tablets $(n=58)$-are compared with the lowest quartile, who consumed 0 tablets $(n=50)$. Highest quartile inpatient opioid users were more likely to be Black $(p=0.006)$. The number of tablets consumed as an inpatient were not related to mode of delivery (spontaneous

Table 1 Sociodemographics of patients who underwent vaginal delivery by quartile of inpatient opioid usage

\begin{tabular}{|c|c|c|c|}
\hline & $\begin{array}{l}\text { Highest quartile } \\
\text { (three tablets or more) } \\
(n=58)\end{array}$ & $\begin{array}{l}\text { Lowest quartile (0 tablets) } \\
(n=50)\end{array}$ & $p$-Value \\
\hline Maternal age at delivery (median [SD]) years & $29(25,32)$ & $32.5(26,35)$ & 0.03 \\
\hline \multicolumn{4}{|l|}{ Race/ethnicity (n [\%]) } \\
\hline White & $17(29.3)$ & $13(26.0)$ & \multirow[t]{3}{*}{0.006} \\
\hline Black & $39(67.2)$ & $25(50.0)$ & \\
\hline Other & $2(332.5)$ & $12(24.0)$ & \\
\hline \multicolumn{4}{|l|}{ Insurance type (n [\%]) } \\
\hline Private insurance & 15 (25.9) & $18(36.0)$ & \multirow[t]{3}{*}{0.2} \\
\hline Public insurance & $42(72.4)$ & $29(58.0)$ & \\
\hline Other & $1(1.7)$ & $3(6.0)$ & \\
\hline Maternal BMI (median [IQR]) & $31.6(26.7,36.2)$ & $29.8(24.8,36.6)$ & 0.6 \\
\hline \multicolumn{4}{|l|}{ Type of delivery (n [\%]) } \\
\hline Spontaneous & $52(89.7)$ & $48(96.0)$ & \multirow[t]{3}{*}{0.2} \\
\hline Vacuum assisted & $3(5.2)$ & $2(4.0)$ & \\
\hline Forceps assisted & $3(5.2)$ & $0(0.0)$ & \\
\hline Type of laceration (n [\%]) & & (missing 1 from lowest quartile) & \\
\hline Intact & $28(48.3)$ & $24(49.0)$ & \multirow[t]{6}{*}{0.1} \\
\hline 1st degree & $3(5.2)$ & $8(16.3)$ & \\
\hline 2nd degree & $12(20.7)$ & $13(26.5)$ & \\
\hline 3rd degree & $1(1.7)$ & $1(2.0)$ & \\
\hline 4th degree & $1(1.7)$ & $0(0.0)$ & \\
\hline Other (periurethral, sulcal) & $13(22.4)$ & $3(6.1)$ & \\
\hline \multicolumn{4}{|l|}{ Complications (n [\%]) } \\
\hline None & $43(74.1)$ & $40(80.0)$ & \multirow[t]{4}{*}{0.08} \\
\hline BTL & $14(24.1)$ & $5(10.0)$ & \\
\hline Cervical laceration & $0(0.0)$ & $2(4.0)$ & \\
\hline Other (episiotomy, IUFD, DVT, trauma) & $1(1.7)$ & $3(6.0)$ & \\
\hline \multicolumn{4}{|l|}{ Discharge opioid subscribing provider (n [\%]) } \\
\hline Intern & $23(39.7)$ & $15(30.0)$ & \multirow[t]{3}{*}{0.2} \\
\hline Resident & $23(39.7)$ & $28(56.0)$ & \\
\hline Attending & $12(20.7)$ & $7(14.0)$ & \\
\hline
\end{tabular}

Abbreviations: BTL, bilateral tubal ligation; DVT, deep venous thrombosis; IQR, interquartile range; IUFD, intrauterine fetal death; SD, standard deviation. 
Table 2 Multivariate regression of number of tablets prescribed at discharge by quartile of inpatient opioid usage after vaginal delivery

\begin{tabular}{|l|l|l|l|}
\hline $\begin{array}{l}\text { \# Oxycodone 5-mg tablets } \\
\text { prescribed at discharge }(\boldsymbol{n}[\%])\end{array}$ & $\begin{array}{l}\text { Highest } \\
\text { quartile }(\boldsymbol{n}=\mathbf{5 8})\end{array}$ & $\begin{array}{l}\text { Lowest quartile (0 tablets) } \\
(\boldsymbol{n}=\mathbf{5 0})\end{array}$ & Unadjusted OR \\
\hline 0 & $31(53.5)$ & $34(68.0)$ & $0.91(0.56-1.48)$ \\
\hline $1-9$ & $1(1.7)$ & $5(10.0)$ & $0.22(0.02-1.98)$ \\
\hline $10-19$ & $16(27.6)$ & $7(14.0)$ & $2.51(0.91-6.90)$ \\
\hline $20-29$ & $6(10.3)$ & $4(8.0)$ & $1.65(0.42-6.38)$ \\
\hline$\geq 30$ & $4(6.9)$ & $0(0.0)$ & - \\
\hline Excluding women who underwent BTL & \multicolumn{3}{|l|}{} \\
\hline $\begin{array}{l}\text { \# Oxycodone 5-mg tablets prescribed } \\
\text { at discharge (n [\%]) }\end{array}$ & Highest quartile $(n=44)$ & $\begin{array}{l}\text { Lowest quartile (0 tablets) } \\
(n=45)\end{array}$ & Unadjusted OR \\
\hline 0 & $27(61.4)$ & $33(73.3)$ & $0.82(0.49-1.36)$ \\
\hline $1-9$ & $1(2.3)$ & $3(6.7)$ & $0.41(0.040-4.14)$ \\
\hline $10-19$ & $9(20.5)$ & $5(11.1)$ & $2.20(0.66-7.35)$ \\
\hline $20-29$ & $4(9.1)$ & $4(8.9)$ & $1.22(0.28-5.35)$ \\
\hline$\geq 30$ & $3(6.8)$ & $0(0.0)$ & - \\
\hline
\end{tabular}

Abbreviations: BTL, bilateral tubal ligation; OR, odds ratio.

or operative VD), degree of perineal laceration, complications such as cervical laceration or BTL, or discharge prescribing physician. As shown in -Table 2, multivariate analysis demonstrated no association between inpatient opioid usage quartile and the number of tablets prescribed at discharge. In a sensitivity analysis excluding patients who underwent BTL, these findings were unchanged.

\section{Cesarean Delivery}

Of the 268 patients who had a $\mathrm{CD}$, the median number of oxycodone 5-mg tablets consumed during the inpatient postoperative period was seven tablets (IQR $2.5,12$ ), and $94.7 \%$ (254/268) of women received an oral opioid prescription at discharge. The median number of oxycodone 5-mg tablets prescribed among those receiving a prescription at discharge was 20 tablets (IQR 20,21). Of the 14.6\% (39/268) patients who did not consume any opioid tablets in the hospital after $C D, 89.7 \%$ (35/39) received a narcotic prescription at discharge, with a median of 20 oxycodone $5-\mathrm{mg}$ tablets (IQR 20, 20).

In - Table 3, the sociodemographic characteristics of top quartile of post-CD inpatient opioid users-who consumed greater than or equal to 12 tablets $(n=74)$-are compared with the lowest quartile, who consumed less than 2.5 tablets $(n=67)$. Highest quartile of inpatient opioid users were more likely to be Black $(p=0.004)$. Patients who stayed until postoperative day 4 were more likely to be in the highest quartile of inpatient opioid users when compared with women who were discharged either on or after postoperative day 5 or on less than postoperative day $3(p=0.02)$. There was no association between inpatient opioid usage and type of insurance or BMI. - Table 4 presents analyses on our primary outcome. There was no association between inpatient opioid usage after $\mathrm{CD}$ and the number of tablets prescribed at discharge, even after adjusting for race and day of discharge. In a sensitivity analysis excluding patients who were discharged after postoperative day 5 , the findings were unchanged.

\section{Discussion}

In this retrospective study, there was no correlation between inpatient opioid consumption and the amount of opioids prescribed at time of discharge after vaginal or CD. Furthermore, postpartum patients within our academic institution received enough oral opioids to last up to 8 days after delivery, exceeding the recommendation of 4 to 7 days of acute pain treatment as per the CDC, regardless of inpatient opioid consumption. Our results also suggest that implementation of standard prescribing after $C D$ is potentially leading to overprescribing; while subjective provider prescribing after VD also leads to overprescribing lending to a need for employing more applicable methodology at the time of discharge.

The median amount of 5-mg oxycodone tablets prescribed was 15 and 20 among those who received a discharge prescription after $V D$ and $C D$, respectively. This is despite the median inpatient opioid usage being 1 tablet (IQR $0-3$ ) and 7 tablets (IQR 2.5-12) for vaginal and cesarean deliveries, respectively. One potential explanation of such difference between inpatient usage and opioids prescribed at discharge is that providers may be accustomed to using a "one size fits all" prescribing protocol after vaginal and cesarean deliveries, which does not incorporate inpatient opioid use into discharge prescribing protocols. This is perhaps best demonstrated through those who had a VD and consumed no postdelivery oral opioids receiving a median of 10 (IQR 5-16.25) tablets at time of discharge. For cesarean sections, nonopioid users in the postoperative setting had a median of 20 tablets (IQR 20-21) prescribed at discharge, the same number for 
Table 3 Sociodemographics of patients who underwent cesarean delivery by quartile of inpatient opioid usage

\begin{tabular}{|c|c|c|c|}
\hline & $\begin{array}{l}\text { Highest quartile ( } \geq 12 \text { tablets) } \\
(n=74)\end{array}$ & $\begin{array}{l}\text { Lowest quartile ( }<2.5 \text { tablets) } \\
(n=67)\end{array}$ & $p$-Value \\
\hline Maternal age at delivery (median [SD]) years & $30(26,33)$ & $30(24,35)$ & 0.9 \\
\hline \multicolumn{4}{|l|}{ Race/Ethnicity (n [\%]) } \\
\hline White & $25(33.8)$ & $40(59.7)$ & \multirow[t]{3}{*}{0.004} \\
\hline Black & $40(54.1)$ & $25(37.3)$ & \\
\hline Other & $9(12.2)$ & $2(3.0)$ & \\
\hline \multicolumn{4}{|l|}{ Insurance type (n [\%]) } \\
\hline Private insurance & $45(60.8)$ & $44(65.7)$ & \multirow[t]{3}{*}{0.4} \\
\hline Public insurance & $27(36.5)$ & $23(34.3)$ & \\
\hline Other & $2(2.7)$ & $0(0.0)$ & \\
\hline Maternal BMI (median [IQR]) & $33.8(29.2,39.8)$ & $32.3(26,6,39.2)$ & 0.3 \\
\hline \multicolumn{4}{|l|}{ Day of discharge (n [\%]) } \\
\hline PPD 3 or less & $25(33.8)$ & $38(56.7)$ & \multirow[t]{3}{*}{0.02} \\
\hline PPD 4 & $43(58.1)$ & $26(38.8)$ & \\
\hline PPD 5 or more & $6(8.1)$ & $3(4.5)$ & \\
\hline \multicolumn{4}{|l|}{ Discharge opioid subscribing provider (n [\%]) } \\
\hline Intern & $38(51.4)$ & $37(55.2)$ & \multirow[t]{3}{*}{0.9} \\
\hline Resident & $22(29.7)$ & $19(28.4)$ & \\
\hline Attending & $14(18.9)$ & $11(16.4)$ & \\
\hline
\end{tabular}

Abbreviations: IQR, interquartile range; PPD, PPD, postpartum day; SD, standard deviation.

Table 4 Multivariate regression of number of tablets prescribed at discharge by quartile of inpatient opioid usage after cesarean delivery

\begin{tabular}{|c|c|c|c|c|}
\hline $\begin{array}{l}\text { \# Oxycodone } 5 \text {-mg tablets } \\
\text { prescribed at discharge ( } n \text { [\%]) }\end{array}$ & $\begin{array}{l}\text { Highest quartile } \\
(\geq 12 \text { tablets }) \\
(n=74)\end{array}$ & $\begin{array}{l}\text { Lowest quartile } \\
(<2.5 \text { tablets }) \\
(n=67)\end{array}$ & Unadjusted OR & Adjusted $\mathrm{OR}^{\mathrm{a}}$ \\
\hline 0 & $0(0.0)$ & $7(10.5)$ & - & - \\
\hline $1-10$ & $0(0.0)$ & $3(4.5)$ & - & - \\
\hline $11-20$ & $46(62.2)$ & $40(59.7)$ & $1.11(0.77-1.42)$ & $1.04(0.85-1.32)$ \\
\hline $21-30$ & $26(35.1)$ & $16(23.9)$ & $1.41(0.67-3.00)$ & $1.68(0.75-3.74)$ \\
\hline$\geq 31$ & $2(2.7)$ & $1(1.5)$ & $1.74(0.15-19.90)$ & $1.85(0.14-23.84)$ \\
\hline \multicolumn{5}{|l|}{ Excluding DC after PPD 5} \\
\hline $\begin{array}{l}\text { \# Oxycodone 5-mg tablets } \\
\text { prescribed at discharge (n [\%]) }\end{array}$ & $\begin{array}{l}\text { Highest quartile } \\
(\geq 12 \text { tablets }) \\
(n=68)\end{array}$ & $\begin{array}{l}\text { Other ( }<12 \text { tablets }) \\
(n=64)\end{array}$ & Unadjusted OR & Adjusted $\mathrm{OR}^{\mathrm{b}}$ \\
\hline 0 & $0(0.0)$ & $7(10.9)$ & - & - \\
\hline $1-10$ & $0(0.0)$ & $3(4.7)$ & - & - \\
\hline $11-20$ & $42(61.8)$ & $38(59.4)$ & $1.10(0.72-1.48)$ & $1.05(0.82-1.34)$ \\
\hline $21-30$ & $25(36.8)$ & $15(23.4)$ & $1.51(0.69-3.28)$ & $1.64(0.73-3.69)$ \\
\hline$\geq 31$ & $1(1.5)$ & $1(1.6)$ & $0.91(0.05-14.97)$ & $0.69(0.38-12.69)$ \\
\hline
\end{tabular}

Abbreviations: DC, discharge; OR, odds ratio; PPD, postpartum day.

${ }^{a}$ Adjusted for race and day of discharge.

${ }^{b}$ Adjusted for race.

routine postoperative oral opioid users. While the IQR after $C D$ suggests less of a variation of opioid prescribing, the median number of tablets consumed as an inpatient is still substantially less. Given it has been demonstrated that after delivery approximately 3.86 million women each year are at risk for continued opioid use, it is imperative to bridge the gap between inpatient usage and opioid prescription at discharge. ${ }^{4}$ One shared decision making study by Prabhu et al suggested one approach to this dilemma, and implemented shared decision making among 51 women after $C D$, 
which showed $50 \%$ decrease in opioids prescribed postoperatively, but still demonstrated median 20 tablets prescribed after cesarean section after shared decision making. ${ }^{11}$ This suggests that additional implementation of review of inpatient opioid usage at time of discharge could more accurately depict opioid consumption to guide adequate discharge opioid prescribing after vaginal and $\mathrm{CD} .{ }^{9}$

Our finding that the number of tablets prescribed at discharge was not associated with inpatient opioid usage supports and adds to the current literature, which remains limited in quantifying inpatient opioid usage after delivery. For example, Badreldin et al found there was no association between amount of tablets prescribed at discharge and median MME consumed in final 24 hours after delivery for both vaginal and cesarean deliveries. ${ }^{9}$ Even further, they also identified that nonopioid users could benefit most from utilizing inpatient opioid consumption to direct postpartum discharge prescriptions. This study appreciated 45.7 and $18.5 \%$ of nonopioid using patients in the last 24 hours until discharge received an opioid prescription after vaginal and $\mathrm{CD}$, respectively. ${ }^{10}$ Our retrospective study supported these findings within a different patient population: our study population was comprised predominantly of Black women with a high proportion of women with public insurance whereas the prior study was comprised mostly of White women with private insurance. Both studies suggest evaluating inpatient opioid usage as a tool to tailor postpartum discharge prescribing after delivery.

This study had many strengths. First, our institution has a centralized electronic medical record, which allowed us to not only quantify number of tablets consumed during the entire inpatient postpartum stay, but also exclude women who had a prior history of opioid use disorder. Second, all data were abstracted from the medical record by physicians, increasing the accuracy of our findings. Third, we included all eligible women during a prespecified time period, which limits the risk of selection bias. Additionally, despite the period of our included patients starting in July at the time of matriculation of newer intern physicians, the discharge prescribing physicians were evenly distributed between intern, resident physician, and attending physician. However, the study is not without limitations. First, our study is retrospective in nature and was conducted at a single institution, which may limit the generalizability of our findings. Second, we were unable to incorporate patient-reported pain scores during inpatient hospitalization. Though these subjective pain measurements may have differed between lowest and highest inpatient opioid users -which could affect the amount of tablets prescribed at discharge-they are inconsistently reported in the patient medical record, particularly on or after postpartum day 2 or postoperative day 3. Lastly, we were not able to perform multivariate regression analysis for VD due to smaller sample size, which can limit identifying potential confounders.

In sum, our study demonstrated that the amount of opioids prescribed at discharge had no association with the amount of opioids consumed during inpatient postpartum stay despite cofounders. Based on the CDC's recommendation of acute pain treatment, patients are likely to have the majority of the acute pain episode while inpatient and therefore tailoring patient's discharge prescription based on inpatient usage can help minimize overprescribing while providing adequate pain control. Additional prospective studies implementing prescribing practices based on inpatient usage and pain scores along with quantifying post discharge opioid usage are warranted to help eliminate overprescribing while maximizing adequate pain control, and to help establish national standards in postpartum opioid prescribing.

\section{Authors' Contribution}

T.M. contributed toward data collection (tyler.mckinnish@wustl.edu). E.C. managed project oversight and manuscript reviewing (ebcarter@wustl.edu). A.L. handled project oversight, statistical analysis, and manuscript reviewing (aklew@gmail.com).

\section{Note}

Presented at: The Annual Meeting of the Society for Maternal-Fetal Medicine (SMFM), The Pregnancy Meeting $^{\mathrm{TM}}$, February 3-8, 2020, Grapevine, Tx.

\section{Conflict of Interest}

None declared.

Acknowledgments

No financial or funding disclosures.

\section{References}

1 Seth P, Scholl L, Rudd RA, Bacon S. Overdose deaths involving opioids, cocaine, and psychostimulants-United States, 20152016. MMWR Morb Mortal Wkly Rep 2018;67(12):349-358

2 Peahl AF, Dalton VK, Montgomery JR, Lai YL, Hu HM, Waljee JF. Rates of new persistent opioid use after vaginal or cesarean birth among US women. JAMA Netw Open 2019;2(07):e197863

3 Prabhu M, Garry EM, Hernandez-Diaz S, MacDonald SC, Huybrechts KF, Bateman BT. Frequency of opioid dispensing after vaginal delivery. Obstet Gynecol 2018;132(02):459-465

4 Bateman BT, Cole NM, Maeda A, et al. Patterns of opioid prescription and use after cesarean delivery. Obstet Gynecol 2017;130 (01):29-35

5 ACOG Committee Opinion No. ACOG Committee Opinion No. 742 summary: postpartum pain management. Obstet Gynecol 2018; 132(01):252-253

6 Alcántara Montero A, Sánchez Carnerero CI, Ibor Vidal PJ, Alonso Verdugo A. CDC guidelines for prescribing opioids for chronic pain. Semergen 2017;43(04):e53-e54

7 Dowell D, Haegerich TM, Chou R. CDC guideline for prescribing opioids for chronic pain-United States, 2016. MMWR Recomm Rep 2016;65(01):1-49

8 Badreldin N, Grobman WA, Yee LM. Inpatient opioid use after vaginal delivery. Am J Obstet Gynecol 2018;219(06):608.e1-608.e7

9 Osmundson SS, Schornack LA, Grasch JL, Zuckerwise LC, Young JL, Richardson MG. Post discharge opioid use after cesarean delivery. Obstet Gynecol 2017;130(01):36-41

10 Badreldin N, Grobman WA, Chang KT, Yee LM. Opioid prescribing patterns among postpartum women. Am J Obstet Gynecol 2018; 219(01):103.e1-103.e8

11 Prabhu M, McQuaid-Hanson E, Hopp S, et al. A shared decisionmaking intervention to guide opioid prescribing after cesarean delivery. Obstet Gynecol 2017;130(01):42-46 